\title{
Gradhiva
}

GRADHIV

Revue d'anthropologie et d'histoire des arts

22 | 2015

Cosmos

\section{Jean-Charles Depaule, avec la collaboration de Jean-Luc Arnaud, À travers le mur}

Marseille, Parenthèses, 2014

Jean-Philippe Garric

\section{OpenEdition}

Journals

Édition électronique

URL : http://journals.openedition.org/gradhiva/3102

DOI : $10.4000 /$ gradhiva.3102

ISSN : 1760-849X

\section{Éditeur}

Musée du quai Branly Jacques Chirac

Édition imprimée

Date de publication : 1 octobre 2015

Pagination : 224-225

ISBN : 978-2-35744-092-0

ISSN : 0764-8928

\section{Référence électronique}

Jean-Philippe Garric, « Jean-Charles Depaule, avec la collaboration de Jean-Luc Arnaud, À travers le mur », Gradhiva [En ligne], 22 | 2015, mis en ligne le 18 septembre 2015, consulté le 24 septembre 2020. URL : http://journals.openedition.org/gradhiva/3102 ; DOI : https://doi.org/10.4000/gradhiva. 3102

Ce document a été généré automatiquement le 24 septembre 2020

(c) musée du quai Branly 


\section{Jean-Charles Depaule, avec la collaboration de Jean-Luc Arnaud, À} travers le mur

Marseille, Parenthèses, 2014

Jean-Philippe Garric

\section{RÉFÉRENCE}

Jean-Charles Depaule, avec la collaboration de Jean-Luc Arnaud, À travers le mur.

Marseille, Parenthèses, 2014, 220 p. 


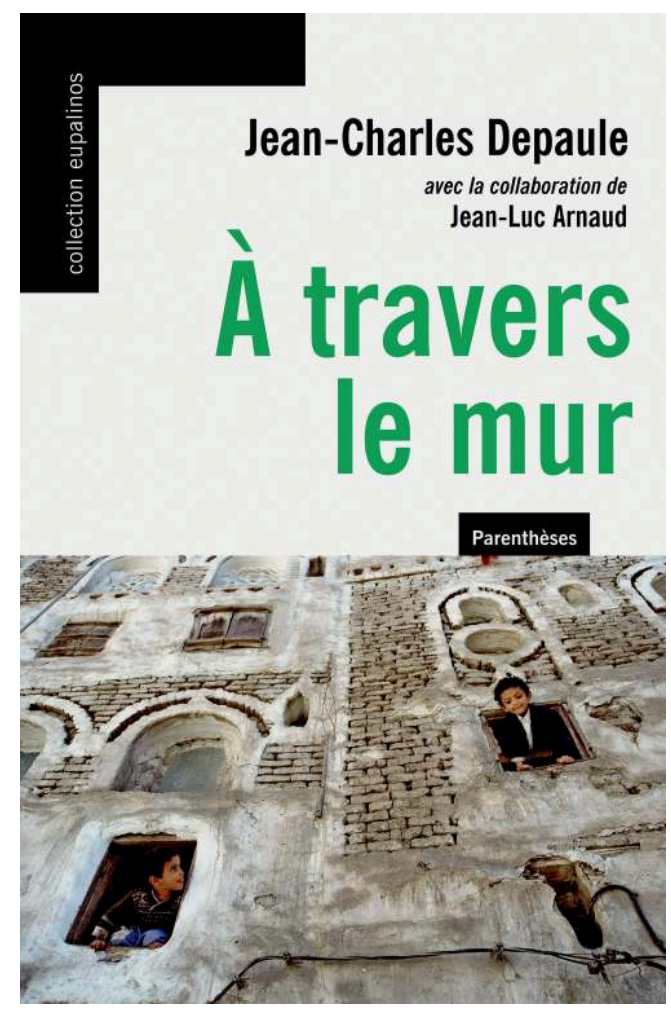

Publié pour la première fois en 1985 dans la collection Alors du Centre de création industrielle, centre Georges-Pompidou, cet ouvrage est le fruit d'une collaboration entre son auteur principal, Jean-Charles Depaule, qui est à la fois chercheur en sciences humaines, poète et traducteur, et Jean-Luc Arnaud, également chercheur en sciences humaines, qui signe les nombreux dessins d'architecture publiés en appui au texte. Si elle prend aujourd'hui, à la lumière de l'actualité, non pas une signification nouvelle, mais une acuité et un relief particuliers, cette nouvelle version est bien plus qu'une simple réédition : c'est le même livre avec trente ans de recul et d'enrichissements.

2 Le mur y est donné pour la frontière qu'il constitue entre l'espace de l'habitation et celui de la ville. Mais cette dualité, loin de se résumer à une opposition binaire entre espace privé et espace public, ou espace domestique et espace urbain, est au contraire analysée dans toute son épaisseur au fil de l'ouvrage, qui en décline les multiples facettes pour mettre en évidence la complexité et l'ambiguïté des pratiques que cet entre-deux organise ou permet d'organiser. Le propos interroge en effet ce que le mur sépare et ce qu'il réunit par les traversées qu'autorisent, en leur donnant lieu et forme, les fenêtres, les portes, les porches, les balcons et toutes les déclinaisons du percement laissant passer, filtrer, percoler, de façon plus ou moins libre ou contrôlée, individuelle ou ritualisée, intense ou raréfiée, les corps et les regards, l'air et la lumière.

Le travail relève d'une anthropologie littéraire de l'espace, ou peut-être plus exactement de l'anthropologie d'un objet architectural complexe et pluriel: le mur de l'habitation dans les "villes de l'Orient arabe", Le Caire, Damas, Alep, Sanaa ou Beyrouth. Il est aussi multiple que son auteur, anthropologue, poète et traducteur, et dont la démarche est aussi en partie liée aux travaux collectifs menés dans le contexte des écoles d'architecture dans les années 1970-1980. Revendiquant à chaque instant la subjectivité de l'expérience et celle du narrateur, l'ensemble n'a rien du pensum ou d'une démonstration académique. Il mobilise à la fois une observation personnelle 
fondée sur une familiarité avec son objet - issue, on l'imagine aisément, d'une fréquentation et d'une pratique récurrentes des édifices, des rues et des centres urbains évoqués - et de nombreux extraits de textes littéraires, récits de voyage, romans et poèmes, souvent traduits par l'auteur, et des photographies, à quoi s'ajoute un nombre important de dessins d'ensemble ou de détail.

Parfois tracées aux instruments, plus souvent dessinées à la main, dans un style qui n'est pas sans rappeler le graphisme des manuels d'architecture de la contre-culture des années 1970, ces illustrations claires et précises développent une approche parallèle à celle du texte plutôt qu'étroitement inféodée à son fil conducteur. Elles informent moins la lecture, dont le déroulé se suffit à lui-même, qu'elles ne confirment, s'ajoutant aux nombreux extraits répartis dans l'ouvrage sous forme d'encadrés, la possibilité de parcourir autrement le volume, d'une façon moins linéaire, par la flânerie et le feuilletage.

Plutôt qu'une hypothèse principale filée tout du long, ce sont plusieurs thèmes qui sont développés successivement à propos de cette traversée, de cette porosité du mur, l'auteur invitant le lecteur, pour ces visites virtuelles, à suivre par étapes la pluralité de son propre regard. Nous en retiendrons trois.

6 Le premier thème repose sur le rapprochement du rôle à la fois protecteur et enfermant du mur de la maison et de celui du vêtement des femmes. Là le moucharabieh et ses divers raffinements, permettant l'occultation maîtrisée des baies, est présenté comme un équivalent du "vêtement à fonction de voile", par les contraintes qu'il induit, mais également par la liberté de voir sans être vue et par le potentiel de transgression et de détournement qu'il recèle. Les pages consacrées à cette question sont à la fois teintées d'une critique de la privation de liberté et d'une séduction de l'interdit un brin romantique et orientaliste. Toutefois, elles peuvent peut-être contribuer à mettre à distance les polémiques, les dérives et les aigreurs contemporaines en enrichissant la question de ses dimensions plurielles liées aux diversités sociales et historiques qui la déterminent.

7 Le deuxième thème concerne la question des seuils. Seuils des maisons, mais aussi 'ataba, seuil présent à l'entrée de chaque pièce principale, marqué par des dispositifs spatiaux comme une pierre de seuil ou un changement dans le motif du dallage, et qui contribue à ritualiser la réception des étrangers ou la pratique du deuil. Mais une attention particulière est aussi accordée aux voies secondaires en impasse, nommées hâra au Caire : des espaces dont le caractère public est restreint par des signes, par exemple du linge étendu à l'entrée, entravant en partie la circulation des personnes, ou la présence d'un tas d'ordures à l'extérieur qualifiant symboliquement un dedans et un dehors. Diversement investies, appropriées suivant les heures de la journée, les jours de l'année, ou au rythme des fêtes et des cérémonies marquant la vie des individus et celle de la communauté, elles sont le lieu d'une activité domestique et familière essentiellement féminine, et d'un partage, jusqu'à l'impudeur, d'une part d'intimité.

8 Le troisième aspect, moins développé bien que récurrent, est celui du rythme des jours, marqués par la succession des activités, mais aussi par le départ puis le retour des hommes. L'ensemble illustre une pratique profondément genrée de l'espace de la ville et de l'habitat, non pas de façon systématique mais par petites touches et par une suite d'anecdotes, qui sont comme autant d'ornements du discours.

On ne peut toutefois s'empêcher de s'interroger sur la façon dont l'ouvrage traite, ou plutôt ne traite pas, de la chronologie. Depaule ne se veut l'historien ni de son objet, ni 
de l'évolution de son propre rapport à l'objet. L'un et l'autre, pourtant, se déploient dans une double durée très souvent sous-jacente au propos. La première épaisseur temporelle concerne l'évolution des villes et des modes de vie observés, entre les exemples anciens pas véritablement situés dans le temps et présentés comme des situations presque intemporelles, évoqués notamment à travers des témoignages littéraires ou la description de dispositifs bâtis hérités du passé, et les comportements actuels observés dans des constructions récentes. Le second intervalle chronologique est celui de la sédimentation et de la transformation du regard de l'auteur lui-même entre les années 1970 et aujourd'hui. Cette absence d'une construction historique prive le lecteur, dans un cas des moyens de comprendre dans quelle mesure la pluralité des situations observées participe d'un processus évolutif ou d'une diversité simultanée, dans l'autre d'une mise à distance et d'une amorce de bilan réflexif. Reste ainsi ouverte la question, pourtant présente en filigrane, de la longue durée des pratiques au regard de leurs mutations. Sans doute pourrait-on faire valoir que plusieurs temps s'entremêlent, celui des époques de production très diverses des quartiers anciens et des quartiers récents, celui de l'origine des types architecturaux à l'œuvre, celui aussi de pratiques et de règles sociales ou religieuses dont les évolutions, selon les villes et les groupes sociaux concernés, ne sont ni homogènes ni linéaires. Il n'en demeure pas moins que cette double historicité, celle de la mutation des pratiques de l'espace dans les villes concernées depuis quarante ans et celle du rapport de certains intellectuels français à ce terrain, pendant le même laps de temps, n'est ici présente que d'une façon qu'on peut juger trop incidente.

10 Cela n'empêche pas pourtant À travers le mur d'éclairer notre temps à partir de celui, moins défensif et moins comptable, où il parut pour la première fois : une époque où la matérialité des pratiques physiques de l'espace n'était pas encore parasitée par la circulation des individus dans les espaces virtuels, où la notion de mur n'avait pas d'avatar dans le monde des réseaux sociaux, ce qui laissait à la rareté des regards brûlants filtrant au travers des moucharabiehs une autre intensité.

11 C'était un temps aussi où le chemin de Damas demeurait une promesse de réinvention personnelle et de refondation intellectuelle; où l'idée de ville orientale rimait davantage avec culture urbaine partagée, entre héritages méditerranéens et romanisés communs et diversité des pratiques sociales, spatiales, religieuses et culturelles, qu'avec un horizon de conflits, de destructions massives et d'horreur. C'étaient des années où les architectes contemporains et les écoles d'architecture françaises, en quête de contre-modèles et d'une compréhension plus fine des pratiques de l'habiter, curieux d'altérité, entre fascination pour les architectures arabo-musulmanes et anticolonialisme, choisissaient de conduire leurs étudiants vers d'autres horizons d'observation et d'apprentissage au lieu de les formater suivant les injonctions du marché du travail. Enfin, À travers le mur nous rappelle qu'à une époque, à la fois si proche et lointaine, un anthropologue écrivain, conjuguant en virtuose flâneries architecturales et dérives poétiques, a passé des heures nombreuses, dans la torpeur des nuits d'été et l'ombre épaisse des terrasses, à voler, dans les entrebâillements, quelques vues plongeantes sur les corps au repos et les intimités, solitaires ou matrimoniales, plutôt qu'à compiler, sans rire, des indicateurs de performance et des rapports d'évaluation. 


\section{AUTEURS}

JEAN-PHILIPPE GARRIC

garric-negre@wanadoo.fr 